\title{
Análisis comparativo de la tributación empresarial en los países de la zona euro durante el período 2000-2013
}

\section{Rafael Molina Llopis, Antonio Barberá Martí}

\author{
Universidad de Valencia \\ Departamento de Contabilidad \\ Grupo Elastic Confort (España) \\ Responsable de Contabilidad
}

Tradicionalmente, algunos países han utilizado el impuesto sobre los beneficios empresariales como una vía para, partiendo de unos tipos relativamente elevados, fomentar determinadas actividades mediante incentivos o bonificaciones fiscales que permitirían reducir considerablemente esta carga fiscal empresarial. En cambio, otros Estados han optado por reducir directamente la tasa de impuestos, sin tener en cuenta los incentivos fiscales, acercando la tributación nominal a la efectiva.

Esta investigación pretende analizar las divergencias existentes en la tributación por el impuesto sobre sociedades entre los distintos países de la eurozona para el período 2000-2013, observando las diferencias entre los tipos nominales y efectivos de las empresas cotizadas. De esta manera, se sitúa en el contexto actual la idea de la Comisión Europea debe adoptar un impuesto de sociedades común, y determinar qué países ejercen una mayor presión fiscal sobre los resultados de sus empresas.

Palabras clave: presión fiscal, tipo impositivo efectivo, empresas cotizadas, eurozona.

\section{Comparative analysis of corporate tax burden in the euro area during 2000-2013}

Traditionally, some countries have been using the Corporate Income Tax as a way of, starting from relatively high nominal tax rates, encourage certain economic activities through incentives or bonuses that allow considerably reduce actual business taxation. In contrast, other member states have chosen to directly lower the tax rate, regardless of other incentives, thus bringing the nominal taxation to effective.

This research aims to analyze the discrepancies in the taxation of corporate income tax between different Eurozone countries for the period 2000-2013, noting the differences between the statutory (STR) and effective tax rates (ETR) of listed companies. In this way, this work locates the idea of the European Commission to adopt a common corporate tax in its current context, determining which countries exercise greater fiscal pressure on the results of their companies.

Keywords: Tax burden, effective tax rate, listed companies, Eurozone. 


\section{Análise comparativa da carga tributária das empresas dos países da zona do euro durante o período 2000-2013}

Tradicionalmente, alguns países com uma elevada taxa nominal utilizam o imposto sobre a renda como uma via para fomentar as atividades econômicas através de incentivos ou bônus que permite reduzir consideravelmente a atual tributação das empresas. Em contrapartida, outros Estados-Membros optaram para diretamente baixar as taxas de impostos, independentemente de outros incentivos, transformando a taxa nominal em efetiva.

Esta pesquisa tem como objetivo analisar as discrepâncias na tributação empresarial entre os diferentes países da zona do euro no período 2000-2013, observando as diferenças entre a taxa nominal e a taxa de imposto efetiva das empresas listadas. Desta forma, este trabalho projeta a noção de que a Comissão Europeia adopte um regime comum para o imposto sobre o rendimento, determinando quais são os países que exercem uma maior carga tributária sobre os resultados de suas empresas.

Palavras-chave: carga tributária, taxa de imposto efetiva, empresas listadas, Zona do Euro.

\section{Introducción}

Desde que en 2008 se empezaran a manifestar las consecuencias negativas de la abrupta finalización de lo que hasta entonces había sido un dilatado período de crecimiento económico, que convergió en una crisis a nivel internacional, prácticamente los gobernantes de todos los países, unos en mayor medida que otros, se vieron obligados a adoptar cambios y reformas en lo que hasta ese momento había sido su patrón de conducta. En líneas generales, como señalan Hemmelgarn y Nicodeme (2010), los gobiernos y bancos centrales de las economías más desarrolladas reaccionaron con una combinación de medidas reguladoras, estímulos fiscales e inyecciones de liquidez.

Transcurrido un tiempo, si bien se observan algunos indicios de mejora, se teme la posibilidad de que la crisis económica que afecta a la Unión Europea dure más de lo previsto debido a los grandes problemas de deuda que, aunque mitigados en los últimos meses por las acciones del Banco Central Europeo (BCE) y las controvertidas políticas de austeridad aplicadas de manera generalizada, todavía persisten en algunos Estados miembros, como Portugal, Irlanda, Italia, Grecia o España. En esta situación de incertidumbre, se siguen planteando medidas orientadas a tratar de superar la crisis de confianza que, aún hoy, sigue afectando a los mercados financieros. En este sentido, uno de los temas recurrentes desde hace años en el seno de la Comisión Europea es la discusión sobre la conveniencia de abordar un proceso de armonización fiscal en el ámbito empresarial, que avance hacia la adopción de un impuesto de sociedades común para todos los estados miembros de la Unión, necesidad que ya se constataba dos décadas atrás en el denominado Informe Ruding ${ }^{1}$.

Entre sus principales conclusiones, este informe señalaba que todos los sistemas fiscales de los Estados miembros practicaban algún tipo de discriminación

1 Se trata del Informe del Comité de Expertos Independientes sobre la Imposición de Sociedades, denominado «Informe Ruding» en referencia al nombre de su presidente, el holandés Onno Ruding. 
entre inversión interior y extranjera, y que los efectos potencialmente distorsionadores de esta situación se verían incrementados por la eliminación de las barreras a la movilidad de capital derivadas de la Unión (Comité de Expertos Independientes sobre la Imposición de Sociedades, 1992). El tema central era si estas distorsiones suponían una amenaza seria para el mercado único y, en caso afirmativo, si podían ser eliminadas simplemente mediante la interacción de las fuerzas del mercado y la competencia entre los regímenes fiscales nacionales, o si era necesaria una actuación a escala comunitaria.

Casi una década más tarde, la Comisión Europea (2001) afirmaba en un interesante trabajo que los sistemas fiscales soportados por las empresas en Europa eran muy ineficientes, por lo que, abandonando la idea inicial de armonizar el impuesto en su conjunto, propuso un sistema alternativo cimentado en la consolidación y reparto de las bases imponibles del impuesto sobre sociedades, conocido como Common Consolidated Corporate Tax Base (CCCTB), desarrollado en la propuesta de directiva presentada por el Consejo en marzo de 2011 (Comisión Europea 2011), todavía pendiente de aprobación definitiva.

Así pues, aunque no se trata de un tema novedoso, la profundización de las diferencias entre los dirigentes europeos como consecuencia de las exigencias económicas derivadas de los rescates y apoyos financieros realizados a diferentes países, algunos de ellos con tipos impositivos claramente inferiores a la media de la Unión, ha vuelto a poner de manifiesto su transcendencia y ha brindado argumentos reforzados a sus defensores, lo que sustenta la oportunidad y relevancia de la presente investigación.

La armonización del impuesto sobre sociedades reduciría la competencia fiscal existente entre los diferentes países, entre otras razones, para atraer nuevas inversiones y evitar posibles deslocalizaciones. Sin embargo, se trata de una cuestión extremadamente compleja, puesto que, con la adopción de la moneda única, perdido el control de la política monetaria, la fiscalidad es, probablemente, la herramienta de política económica de mayor relevancia que todavía conservan los gobiernos, más aún si se considera el acuerdo alcanzado por la Eurocámara en marzo de 2013 —conocido como Two-Pack-, para incrementar el control comunitario sobre los presupuestos generales de los estados de la zona euro a partir del año 2014.

En este sentido, algunos países han estado utilizando el impuesto sobre beneficios como vía para, partiendo de tipos nominales relativamente altos, fomentar determinadas actividades económicas a través de incentivos o bonificaciones que permitían reducir sustancialmente la tributación real de las empresas. En cambio, otros Estados miembros han optado por bajar directamente los tipos tributarios, prescindiendo de otros incentivos; de este modo, han acercado la fiscalidad nominal a la efectiva ${ }^{2}$.

Esta dualidad de sistemas fiscales hace que el tipo nominal (TN) del impuesto sobre sociedades no sea una referencia adecuada para comparar la presión fiscal realmente soportada por las empresas en los distintos países. En esa medida, es necesario definir un indicador alternativo que sea capaz de incorporar

2 Para un estudio más profundo de esta cuestión, puede consultarse los trabajos de Edgerton (2010), Elschner y Vanborren (2009) o Hemmelgarn y Nicodeme (2010), entre otros. 
el impacto de las diferentes deducciones o bonificaciones a las que haya podido acogerse el sujeto pasivo, al que, siguiendo la extensa literatura sobre el tema, denominaremos «tipo impositivo efectivo» (TIE) ${ }^{3}$.

Entre las principales investigaciones que han recurrido a esta variable para analizar la tributación empresarial, dependiendo de la finalidad perseguida en la investigación, siguiendo a Molina (2005), se encuentran tres tipologías de trabajos: los centrados en la comparación fiscal entre distintos países o regiones, los dirigidos a estudiar los efectos sobre la carga fiscal de un cambio en la normativa tributaria y aquellos dedicados a determinar las principales variables económico-financieras que inciden sobre la tributación efectiva. El presente trabajo, enmarcado en la primera de esas categorías, tiene como objetivo principal analizar las divergencias existentes en la tributación por el impuesto sobre sociedades entre los distintos países de la zona euro, con el fin último de cuantificar las diferencias en la presión fiscal que verdaderamente soportan las empresas cotizadas en cada uno de los Estados miembros, estudiando las discrepancias entre los tipos impositivos nominales y efectivos para el período 2000-2013.

Como referencias en esta línea de investigación, en el ámbito europeo, podemos citar los trabajos de Jacobs y Spengel (2000), Buijink, Janssenb y Schols (2002), Nicodème (2007), Romero, Molina y Labatur (2009), Álvarez, Fernández y Martínez (2011), y Lazăr (2013). Fuera del ámbito europeo, cabe mencionar los trabajos de Collins, Kemsley y Shackelford (1995), Rohaya, Nor'Azam y Barjoyai (2008), o Markle y Shackelford (2012), entre otros.

\section{El TIE como medida de la presión fiscal}

Desde que a principios de los años setenta del siglo pasado la Securities and Exchange Commission -agencia federal encargada de la supervisión de los mercados financieros estadounidenses equivalente en España a la Comisión Nacional del Mercado de Valores- exigiera a las empresas cotizadas la presentación de una conciliación entre los tipos impositivos nominales y los efectivamente soportados, el interés por cuantificar estos últimos a fin de evaluar el impacto de las políticas tributarias, $y$, en consecuencia, el verdadero nivel de presión fiscal aplicado por los Estados, ha sido creciente. Ello ha dado lugar a la realización de numerosos estudios que han utilizado como indicador el TIE, aunque con distintas formulaciones para su cálculo en función de los objetivos perseguidos.

Así, básicamente, podemos distinguir entre TIE medio y marginal. El primero, según explican Buijink y otros (2002), mide la parte del resultado contable antes de impuestos, que representa el impuesto pagado en un determinado año — trabajos de carácter retrospectivo-, y es la variable habitualmente utilizada en las investigaciones dirigidas a comparar la tributación efectiva entre diferentes países o colectivos. El TIE marginal, por su parte, calcula la cantidad que el contribuyente deberá pagar por la obtención de una unidad adicional de renta - estudios prospectivos-. Como señalan Devereux y Griffith (2003), este permite determinar el impacto del régimen tributario en un proyecto de inversión futuro, por lo que es el indicador de referencia en las investigaciones dirigidas a evaluar cómo los impuestos afectan a las decisiones empresariales.

\footnotetext{
3 Es ETR por sus siglas en inglés (Effective Tax Rate).
} 
Este trabajo se enfoca en el TIE medio, indicador elegido atendiendo a sus objetivos, dado que, en este, se pretende cuantificar la presión fiscal soportada. Como indica Molina (2005), parece evidente que dicho TIE debe relacionar la carga impositiva con la base gravable que la ha generado. Aunque no existe unanimidad a la hora de definir estas magnitudes, las detalladas revisiones de la literatura especializada realizadas por Plesko (2003) y Molina (2005) muestran que la mayoría de los artículos relevantes en este campo se han decantado por formular el TIE medio como el gasto contable por el impuesto sobre beneficios, corregido en algunos casos por los impuestos diferidos, dividido por el resultado contable antes de impuestos. Esta elección está claramente condicionada por la imposibilidad habitual de disponer de datos fiscales para el análisis, lo que ha dado lugar a que la mayor parte de las investigaciones realizadas estén basadas en las cifras recogidas en los estados financieros de las empresas.

Otra cuestión relevante en este tipo de estudios es la duración del período analizado, debido a que los efectos de la aplicación del método del efecto impositivo para contabilizar el impuesto podrían condicionar los valores del TIE a corto plazo. En este sentido, Dyreng, Hanlon y Maydew (2008) sostienen que analizar la presión fiscal de las compañías tomando el tipo efectivo de un único ańo podría no ser suficiente, pues la carga fiscal soportada en un ejercicio aislado está expuesta a sesgos — por ejemplo, como consecuencia del impacto de una diferencia temporaria de cuantía significativa-, que podrían elevar o reducir el tipo efectivo de manera transitoria, confundiéndose los efectos de un acontecimiento puntual con la posición de la empresa frente a la fiscalidad.

Por todo lo anterior, este trabajo analizará la presión fiscal derivada del impuesto sobre sociedades soportada por las empresas cotizadas de los países de la zona euro, considerando un período de 14 años —-2000 a 2013-. En este marco, se entiende el TIE medio como el cociente entre el gasto por impuesto sobre beneficios y el beneficio antes de impuestos.

\section{Análisis comparativo de la presión fiscal societaria en la zona euro}

Para la realización del estudio, partiendo de la base de datos Osiris (Bureau Van Dijk), se ha confeccionado una muestra con la información contable de las empresas cotizadas radicadas en los países que formaban parte de la eurozona en 2013, en la que se ha eliminado a las sociedades financieras y de seguros (código K en Nace Rev. 2 sección principal) por sus particularidades fiscales, como Buijink y otros (2002), Rohaya y otros (2008), o Álvarez y otros (2011), entre otros. Además, se ha depurado los valores extremos para evitar distorsiones en los resultados eliminando los que se situaban fuera del intervalo $[\mu \pm 2 \sigma]$.

La tabla 1 presenta el número de observaciones de la muestra, organizada por países y años, así como las cuatro agrupaciones de datos realizadas para el desarrollo de los diferentes estudios. La exclusión de Estonia y Malta en $U E M 17 b$ y $U E M b$, subconjuntos muestrales utilizados para el análisis del Tie, responde al reducido número de datos obtenidos en estos países, lo cual podría comprometer la consistencia de los resultados.

Aplicando una metodología descriptiva, el trabajo realiza un análisis de los tipos nominales y efectivos, tanto por países como por su evolución a lo largo del período objeto de estudio.

\section{Resultados}

\subsection{Análisis de la presión fiscal nominal}

El primer estudio realizado es un análisis comparativo de los tipos nominales establecidos en cada uno de los 
países y su evolución a lo largo del espacio objeto de análisis. La tabla 2, partiendo del trabajo publicado por la Comisión Europea (2014), presenta los tipos nominales de los 17 países desde el año 2000 al 2013, así como diferentes cálculos que se comentan a continuación.

Como puede observarse, Alemania (36,0\%) e Italia $(35,6 \%)$ son los países con las medias del período más elevadas. En el lado opuesto, aparecen Chipre (14,8\%) e Irlanda (14,1\%), a más de 20 puntos de distancia. Con respecto a las diferentes agrupaciones realizadas, los valores medios calculados con el conjunto de países miembros en 2013 -UEM17a (28,4\%) y UEM17b (28,3\%) — son superiores a los obtenidos considerando los Estados que pertenecían a la eurozona cada uno de los ańos - UEMa $(29,6 \%)$ y UEMb $(29,4 \%)$ - Esto se debe a la influencia en la media

Tabla 1. Composición de la muestra final por países y años

\begin{tabular}{|c|c|c|c|c|c|c|c|c|c|c|c|c|c|c|c|}
\hline País / Año & 2000 & 2001 & 2002 & 2003 & 2004 & 2005 & 2006 & 2007 & 2008 & 2009 & 2010 & 2011 & 2012 & 2013 & Total \\
\hline Alemania (1999) & 288 & 259 & 248 & 274 & 264 & 316 & 332 & 357 & 324 & 303 & 374 & 386 & 390 & 375 & 4,490 \\
\hline Austria (1999) & 0 & 0 & 0 & 3 & 34 & 46 & 52 & 49 & 39 & 42 & 49 & 59 & 51 & 55 & 479 \\
\hline Bélgica (1999) & 58 & 60 & 58 & 65 & 69 & 81 & 83 & 91 & 75 & 68 & 85 & 81 & 84 & 79 & 1,037 \\
\hline Chipre (2008) & 8 & 32 & 34 & 26 & 6 & 10 & 58 & 71 & 46 & 45 & 42 & 30 & 24 & 20 & 452 \\
\hline Eslovaquia (2009) & 36 & 38 & 42 & 45 & 10 & 11 & 17 & 17 & 15 & 9 & 15 & 20 & 26 & 23 & 324 \\
\hline Eslovenia (2007) & 0 & 0 & 12 & 27 & 12 & 15 & 18 & 21 & 15 & 15 & 14 & 19 & 20 & 13 & 201 \\
\hline España (1999) & 93 & 95 & 82 & 93 & 74 & 81 & 90 & 83 & 76 & 73 & 69 & 74 & 63 & 65 & 1,111 \\
\hline Estonia (2011) & 0 & 0 & 0 & 0 & 4 & 10 & 12 & 12 & 8 & 4 & 8 & 8 & 9 & 10 & 85 \\
\hline Finlandia (1999) & 79 & 78 & 73 & 77 & 73 & 78 & 82 & 81 & 75 & 51 & 71 & 72 & 67 & 61 & 1,018 \\
\hline Francia (1999) & 373 & 359 & 368 & 376 & 326 & 360 & 381 & 410 & 357 & 319 & 376 & 372 & 354 & 332 & 5,063 \\
\hline Grecia (2001) & 100 & 109 & 99 & 120 & 100 & 130 & 144 & 148 & 109 & 96 & 67 & 59 & 53 & 51 & 1,385 \\
\hline Irlanda (1999) & 17 & 16 & 17 & 17 & 17 & 21 & 27 & 30 & 17 & 16 & 27 & 27 & 30 & 28 & 307 \\
\hline Italia (1999) & 81 & 81 & 76 & 85 & 100 & 107 & 104 & 120 & 108 & 91 & 111 & 107 & 104 & 102 & 1,377 \\
\hline Luxemburgo (1999) & 3 & 3 & 4 & 4 & 18 & 18 & 22 & 24 & 23 & 26 & 32 & 32 & 30 & 27 & 266 \\
\hline Malta (2008) & 6 & 4 & 3 & 0 & 2 & 2 & 2 & 11 & 7 & 9 & 7 & 9 & 11 & 11 & 84 \\
\hline Países bajos (1999) & 77 & 71 & 66 & 65 & 70 & 74 & 74 & 77 & 64 & 61 & 90 & 76 & 63 & 75 & 1,003 \\
\hline Portugal (1999) & 27 & 25 & 20 & 23 & 24 & 26 & 33 & 30 & 30 & 33 & 35 & 32 & 28 & 30 & 396 \\
\hline $\mathrm{UEM}_{17 \mathrm{a}^{\mathrm{a}}}$ & 1,246 & 1,230 & 1,202 & 1,300 & 1,203 & 1,386 & 1,531 & 1,632 & 1,388 & 1,261 & 1,472 & 1,463 & 1,407 & 1,357 & 19,078 \\
\hline $\mathrm{UEM}^{17 b^{b}}$ & 1,240 & 1,226 & 1,199 & 1,300 & 1,197 & 1,374 & 1,517 & 1,609 & 1,373 & 1,248 & 1,457 & 1,446 & 1,387 & 1,336 & 18,909 \\
\hline $\mathrm{UEMa}^{\mathrm{c}}$ & 1,096 & 1,156 & 1,111 & 1,202 & 1,169 & 1,338 & 1,424 & 1,521 & 1,365 & 1,257 & 1,464 & 1,463 & 1,407 & 1,357 & 18,330 \\
\hline $\mathrm{UEMb}^{\mathrm{d}}$ & 1,096 & 1,156 & 1,111 & 1,202 & 1,169 & 1,338 & 1,424 & 1,521 & 1,358 & 1,248 & 1,457 & 1,446 & 1,387 & 1,336 & 18,249 \\
\hline
\end{tabular}


de los tipos más reducidos que poseían los nuevos miembros incorporados a la zona euro a lo largo del período analizado (Grecia, Eslovenia, Chipre, Malta, Eslovaquia y Estonia).

En cuanto a la evolución, la presión fiscal nominal ha seguido una tendencia general claramente bajista con la única excepción de Malta, que no ha modificado su tipo impositivo en todo el período: este se ha mantenido estable en el 35\%. Sin embargo, la mala situación de las arcas públicas derivada de la caída de actividad ocasionada por la crisis ha dado lugar a importantes repuntes en los tipos de diferentes países en los últimos años. Así, Francia y Portugal en 2012

Tabla 2. Evolución anual del tipo nominal por países y ańos (\%)

\begin{tabular}{|c|c|c|c|c|c|c|c|c|c|c|c|c|c|c|c|c|}
\hline & 000 & 2001 & 2002 & 003 & 2004 & 2005 & 006 & 2007 & 2008 & 2009 & 2010 & 2011 & 2012 & 2013 & Media & Dif. $\%^{\mathrm{f}}$ \\
\hline & 51.6 & 38.3 & 38.3 & 39.6 & 38.3 & 38.7 & 38.7 & 38.7 & 30.2 & 30.2 & 30.2 & & & 30.2 & & \\
\hline & & 34 & 34 & 34 & 24 & 25 & 25 & 25 & 25 & 25 & 25 & 25 & 25 & 25 & & $-26.47 \%$ \\
\hline & 40.2 & 40.2 & 40.2 & & & 34 & 34 & & & 34 & & & & & & \\
\hline & 35 & 35 & 35 & & & 35 & 35 & & & 30 & & & & & & \\
\hline & & 29 & 29 & & & 26 & & & & 26 & & & & & & \\
\hline & 37.8 & 36.4 & 35.4 & 35.4 & 35.4 & 35 & 34.4 & 34.4 & 34.4 & 34.4 & 34.4 & 34.4 & 36.1 & 36.1 & & \\
\hline & & 20 & 16 & 12.5 & 12.5 & 12.5 & 12.5 & 12.5 & 12.5 & 12.5 & 12.5 & 12.5 & 12.5 & 12.5 & 14.11 & $-47.92 \%$ \\
\hline & & 40.3 & 40.3 & & & & & & & & & & & & & \\
\hline & 375 & 37.5 & 30.4 & 30.4 & 30.4 & 30.4 & 29.6 & 29.6 & 29.6 & 28.6 & 28.6 & 28.8 & 28.8 & & & \\
\hline & 35 & 35 & 34.5 & & & & & 25.5 & & 25.5 & & & & & & $57 \%$ \\
\hline & 35.2 & 35.2 & 33 & & 27.5 & 27.5 & 27.5 & 26.5 & 26.5 & 26.5 & & & 31.5 & 31.5 & 29.96 & \\
\hline & 40 & 37.5 & 35 & 35 & 35 & 32 & 29 & 25 & 35 & 35 & 24 & 20 & 20 & 26 & 30.61 & $-35.00 \%$ \\
\hline & 29 & 25 & 25 & & & 25 & 25 & & & 21 & & & & & & \\
\hline & & 28 & 28 & & & 10 & 10 & 10 & 10 & 10 & & & & 12.5 & & \\
\hline & & 35 & 35 & 35 & 35 & 35 & 35 & 35 & 35 & 35 & & & & & 35 & \\
\hline & 29 & 29 & 25 & & 19 & 19 & 19 & 19 & 19 & 19 & & & & & & \\
\hline Estoniae & 26 & 26 & 26 & 26 & 26 & 24 & 23 & 22 & 21 & 21 & 21 & & 21 & 21 & 23.21 & $-19.23 \%$ \\
\hline $\mathrm{UEM}_{17 \mathrm{a}^{\mathrm{a}}}$ & & 33.02 & 31.77 & 30.39 & 29.58 & 28.11 & 27.68 & 26.82 & 26.30 & 26.18 & 25.62 & & 25.41 & 26.11 & 28.36 & $-24.58 \%$ \\
\hline & 35.17 & 33.36 & 31.94 & 30.38 & 29.46 & 27.93 & 27.51 & 26.60 & 26.07 & 25.94 & 25.31 & 25.02 & 25.07 & 25.86 & 28.26 & $-26.48 \%$ \\
\hline $\mathrm{UEMa}^{\mathrm{c}}$ & 36.42 & 34.87 & 33.43 & 32.56 & 31.91 & 30.41 & 29.88 & 28.46 & 27.14 & 26.51 & 25.91 & 25.37 & 25.41 & 26.11 & 29.60 & $-28.30 \%$ \\
\hline $\mathrm{UEMb}^{\mathrm{d}}$ & 36.42 & 34.87 & 33.43 & 32.56 & 31.91 & 30.41 & 29.88 & 28.46 & 26.58 & 25.94 & 25.31 & 25.02 & 25.07 & 25.86 & 29.41 & $-28.99 \%$ \\
\hline
\end{tabular}

Fuente: Comisión Europea (2014). Elaboración propia

a Países miembros de la zona euro en 2013

b UEM17a menos Estonia, Luxemburgo y Malta

c Países miembros de la zona euro en cada uno año de los años analizados

d UEMa menos Estonia y Malta

e En cursiva, los años en los que el país todavía no formaba parte de la zona euro

$\mathrm{f}$ Variación total del período analizado en porcentaje

(Situada en el punto 4.1) 
y Chipre, Eslovaquia y Grecia en 2013 han elevado sustancialmente sus tipos nominales, hasta 6 puntos porcentuales en el último caso.

A pesar de ello, la relevancia de la reducción de los tipos experimentada en la mayoría de los Estados miembros hace que la presión fiscal nominal media en la eurozona se haya reducido en casi un 30\% a lo largo del período analizado —entre 8,5 y 10,6 puntos porcentuales, dependiendo de los países considerados-. En particular, destacan Chipre $(-56,9 \%)$, Irlanda $(-47,9 \%)$, Alemania $(-41,5 \%)$ y Eslovenia $(-41,4 \%)$, que han rebajado sus tipos nominales por encima del $40 \%$, mientras que, en el lado contrario, además de la mencionada Malta, Francia $(-4,5 \%)$ es el país que menor reducción presenta.

Respecto al camino seguido para estas modificaciones, un análisis detallado de los valores de la tabla 2 pone de manifiesto que no todos los países han sido igual de activos. A lo largo de los ańos estudiados, se han producido 65 modificaciones de tipos nominales - 54 a la baja y 11 incrementos-, casos entre los cuales destacan a Grecia (9), Eslovenia (7), Alemania (5), Francia (5), Luxemburgo (5), Países Bajos (5), Portugal (5), Chipre (4), Italia (4) y Estonia (4). En el caso de España, se observan 2 reducciones —años 2007 y 2008 - , aunque, en realidad, ambas son consecuencia de un único cambio normativo introducido por la Ley 35/2006 que preveía un descenso global de 5 puntos en el tipo general, a implementar por mitades en los 2 períodos impositivos siguientes.

Para finalizar este apartado, cabe subrayar la agresiva rebaja de más de 13 puntos porcentuales realizada por Alemania en el año 2001 para tratar de contener la desaceleración de su economía, así como la decidida política de Irlanda, que, entre 2001 y 2003, redujo su tipo nominal casi a la mitad.

\subsection{Análisis de la presión fiscal efectiva}

En el segundo estudio, se realiza una comparativa de la tributación efectiva de las sociedades, tanto por países como en su evolución a lo largo del período considerado. En la tabla 3, partiendo de los valores medios obtenidos por cada país, se expone el tipo impositivo efectivo de los 15 países (desde el año 2000 al 2013) que presentaban un número de observaciones relevante, así como diferentes cálculos que se comentan a posteriori.

Por un lado, Italia $(35,5 \%)$ y Grecia $(30,6 \%)$ son los países con las medias más elevadas del período. En el lado opuesto, destacan Chipre (14,8\%) e Irlanda $(14,1 \%)$, a más de 15 puntos de distancia. Así, la ligera diferencia encontrada en este período entre las principales agrupaciones - UEM17b (23,9\%) y UEMb $(24,9 \%)$ - indica que los nuevos miembros incorporados a la zona euro a lo largo del período poseían un TIE medio ligeramente inferior al resto de países.

Respecto su evolución, la presión fiscal efectiva ha seguido una tendencia bajista, a pesar de que algunos países han llegado incluso a incrementarlo levemente a lo largo del período. En este sentido, la mayoría de estos incrementos se producen desde el año 2008 y como respuesta de los gobiernos a la crisis económica, sobre todo a través de la eliminación de deducciones e incentivos fiscales que permitían reducir la tributación efectiva. Así, observando el período 2008-2013, se distingue un importante incremento en Eslovaquia, Grecia, Chipre, España, Francia e Italia, todos ellos entre 2 y 5 puntos porcentuales.

No obstante, la importancia de la reducción observada en la mayoría de los países genera que la presión fiscal efectiva media de la eurozona se reduzca en casi un $20 \%$ a lo largo del espacio estudiado —entre 4,8 
y 5,3 puntos porcentuales, dependiendo de los países considerados-. En concreto, Bélgica (-37,2\%), Alemania $(-31,4 \%)$, Países Bajos $(-28,0 \%)$, Eslovaquia $(-26,7 \%)$ y Portugal $(-22,7 \%)$ presentan los mayores recortes, entre 7 y 12 puntos sobre el tipo que poseían al inicio del período. En el lado opuesto, aparecen Luxemburgo $(+34,6 \%)$ y Austria $(+24,5 \%)$, que, junto a España $(+7,0 \%)$, Grecia $(+4,0 \%)$ y Eslovenia $(2,5 \%)$, son los únicos países que han llegado a incrementarlo hasta 5 puntos porcentuales. Para terminar con este epígrafe, cabe mencionar el importante recorte de casi 12 puntos observado en Eslovenia
$(-54,3 \%)$ desde el año 2006 hasta el final del período, justo en los años en que más incrementos del tipo efectivo se han realizado.

\subsection{Análisis de las diferencias entre el tipo nominal y efectivo}

El tercer y último estudio es una comparación entre los resultados obtenidos en los dos apartados anteriores, que contrasta las diferencias entre el tipo nominal y el tipo efectivo, tanto en valores medios como en su evolución, para obtener la influencia de los incentivos fiscales en cada país. La tabla 4 muestra las clasificaciones

Tabla 3. Evolución del tipo impositivo efectivo por países y ańos (\%)

\begin{tabular}{|c|c|c|c|c|c|c|c|c|c|c|c|c|c|c|c|c|}
\hline País / Año & 2000 & 2001 & 2002 & 2003 & 2004 & 2005 & 2006 & 2007 & 2008 & 2009 & 2010 & 2011 & 2012 & 2013 & Media & Dif. $\%^{a}$ \\
\hline Alemania & 36.61 & 35.33 & 33.57 & 33.99 & 32.24 & 30.06 & 29.23 & 28.65 & 27.30 & 27.22 & 24.85 & 26.36 & 25.16 & 25.11 & 29.69 & $-31.43 \%$ \\
\hline Austria & c & c & c & 16.49 & 24.33 & 20.15 & 20.39 & 19.39 & 21.66 & 19.77 & 18.80 & 20.23 & 20.91 & 20.54 & 20.24 & $24.51 \%$ \\
\hline Bélgica & 29.33 & 28.67 & 30.24 & 29.28 & 24.44 & 24.61 & 21.90 & 21.76 & 22.27 & 22.02 & 20.05 & 22.45 & 18.51 & 18.42 & 23.85 & $-37.19 \%$ \\
\hline España & 23.87 & 23.42 & 22.93 & 24.72 & 24.64 & 26.56 & 25.22 & 23.05 & 22.90 & 25.19 & 25.53 & 24.70 & 25.14 & 25.55 & 24.53 & $7.03 \%$ \\
\hline Finlandia & 29.19 & 28.50 & 30.08 & 28.54 & 26.57 & 26.11 & 25.63 & 24.78 & 25.83 & 25.82 & 25.74 & 25.05 & 26.13 & 23.45 & 26.53 & $-19.65 \%$ \\
\hline Francia & 32.5 & 31.76 & 32.16 & 32.09 & 29.56 & 28.40 & 29.40 & 28.52 & 29.04 & 29.16 & 28.84 & 29.89 & 30.60 & 31.11 & 30.22 & $-4.30 \%$ \\
\hline Irlanda & 13.94 & 16.95 & 14.60 & 15.53 & 14.11 & 11.80 & 13.54 & 14.80 & 13.96 & 14.19 & 14.59 & 13.54 & 12.49 & 13.04 & 14.08 & $-6.42 \%$ \\
\hline Italia & 38.57 & 35.54 & 35.15 & 36.25 & 37.18 & 34.69 & 37.14 & 35.72 & 33.15 & 34.41 & 34.22 & 36.22 & 33.45 & 35.73 & 35.53 & $-7.36 \%$ \\
\hline & 15.98 & 15.94 & 18.16 & 17.48 & 24.14 & 17.29 & 18.94 & 22.21 & 21.33 & 22.17 & 16.47 & 19.71 & 22.52 & 21.50 & & $34.57 \%$ \\
\hline Países bajos & 28.56 & 28.97 & 28.13 & 25.62 & 28.01 & 25.68 & 22.16 & 21.36 & 23.43 & 21.26 & 20.21 & 21.39 & 21.90 & 20.55 & 24.09 & $-28.03 \%$ \\
\hline Portugal & 31.09 & 29.72 & 29.08 & 32.50 & 24.41 & 22.36 & 25.44 & 23.40 & 24.76 & 25.74 & 26.69 & 29.01 & 30.24 & 24.02 & 27.03 & $-22.74 \%$ \\
\hline Greciaa & 31.63 & 31.64 & 33.77 & 30.48 & 33.03 & 31.50 & 29.39 & 26.47 & 27.89 & 31.83 & 34.94 & 26.80 & 25.64 & 32.89 & 30.56 & $3.96 \%$ \\
\hline Eslovenia $^{a}$ & $c$ & $c$ & 9.43 & 11.55 & 15.69 & 20.40 & 21.14 & 17.98 & 16.25 & 15.43 & 16.53 & 20.23 & 12.38 & 9.66 & 15.56 & $2.46 \%$ \\
\hline Chipre $^{\mathrm{a}}$ & 19.2 & 24.35 & 17.38 & 15.93 & 10.37 & 13.59 & 11.34 & 11.87 & 14.04 & 11.45 & 11.58 & 13.81 & 12.69 & 18.92 & 14.75 & $-1.51 \%$ \\
\hline Eslovaquia $^{a}$ & 27.18 & 24.35 & 22.31 & 21.29 & 19.67 & 22.50 & 20.98 & 18.83 & 14.67 & 16.78 & 18.76 & 19.76 & 18.77 & 19.91 & 20.41 & $-26.72 \%$ \\
\hline UEM17b & 27.51 & 27.32 & 25.50 & 24.78 & 24.56 & 23.71 & 23.46 & 22.59 & 22.57 & 22.83 & 22.52 & 23.28 & 22.44 & 22.69 & 23.98 & $-17.51 \%$ \\
\hline UEMb & 27.96 & 27.86 & 27.99 & 26.91 & 26.89 & 24.93 & 24.87 & 23.70 & 23.13 & 22.83 & 22.52 & 23.28 & 22.44 & 22.69 & 24.86 & $-18.85 \%$ \\
\hline
\end{tabular}

a En cursiva, los años en los que el país todavía no formaba parte de la zona euro.

b Variación total del período analizado en porcentaje.

c Años que en los que no existen datos analizables

(Situada en el punto 4.2) 
por países, según las medias de ambos tipos estudiados y sus diferencias.

En este sentido, Luxemburgo (36,2\%), Bélgica (32,5\%), y Eslovenia $(31,9 \%)$ son los países que presentan mayores diferencias entre el tipo nominal y el efectivo. En cambio, las menores se encuentran en Italia $(0,1 \%)$ y Grecia $(0,2 \%)$, separadas por más de 30 puntos de distancia de las anteriores.
En lo que respecta a las agrupaciones realizadas, el conjunto de países miembros de la eurozona en cada año —UEMb (15,5\%)— muestra unas diferencias más elevadas que las obtenidas considerando los Estados que pertenecían en 2013 -UEM17b (15,2\%)—. Esto refleja una mayor relevancia de los incentivos fiscales en la tributación empresarial de los antiguos miembros de la zona euro respecto a la de los estados más recientes.

Tabla 4. Clasificación según medias del período y variación entre ellas (\%)

\begin{tabular}{|c|c|c|c|c|c|c|c|c|}
\hline \multicolumn{3}{|c|}{ Ranking $\mathrm{TN}^{\mathrm{a}}$} & \multicolumn{3}{|c|}{ Ranking TIE } & \multicolumn{3}{|c|}{ Ranking (TN - TIE) ${ }^{c}$} \\
\hline 1 & Alemania & 35.96 & 1 & Italia & 35.53 & 1 & Luxemburgo & $36.22 \%$ \\
\hline 2 & Italia & 35.56 & 2 & Grecia & 30.56 & 2 & Bélgica & $32.49 \%$ \\
\hline 3 & Bélgica & 35.33 & 3 & Francia & 30.22 & 3 & Eslovenia & $31.93 \%$ \\
\hline 4 & Francia & 35.29 & 4 & Alemania & 29.69 & 4 & Austria & $28.25 \%$ \\
\hline 5 & Malta & 35 & 5 & Portugal & 27.03 & 5 & España & $24.94 \%$ \\
\hline 6 & España & 32.68 & 6 & Finlandia & 26.53 & 6 & Países Bajos & $18.06 \%$ \\
\hline 7 & Luxemburgo & 30.67 & - & EMUb & 24.86 & 7 & Alemania & $17.44 \%$ \\
\hline 8 & Grecia & 30.61 & 7 & Espańa & 24.53 & - & EMUb & $15.47 \%$ \\
\hline 9 & Portugal & 29.96 & 8 & Países Bajos & 24.09 & - & EMU17b & $15.15 \%$ \\
\hline- & $U E M a$ & 29.6 & - & EMU17b & 23.98 & 8 & Francia & $14.37 \%$ \\
\hline - & $U E M b$ & 29.41 & 9 & Bélgica & 23.85 & 9 & Portugal & $9.78 \%$ \\
\hline 10 & Países Bajos & 29.4 & 10 & Eslovaquia & 20.41 & 10 & Eslovaquia & $5.38 \%$ \\
\hline- & $U E M 17 a$ & 28.36 & 11 & Austria & 20.24 & 11 & Finlandia & $1.23 \%$ \\
\hline- & $U E M 17 b$ & 28.26 & 12 & Luxemburgo & 19.56 & 12 & Chipre & $0.47 \%$ \\
\hline 11 & Austria & 28.21 & 13 & Eslovenia & 15.56 & 13 & Irlanda & $0.21 \%$ \\
\hline 12 & Finlandia & 26.86 & 14 & Chipre & 14.75 & 14 & Grecia & $0.16 \%$ \\
\hline 13 & Estonia & 23.21 & 15 & Irlanda & 14.08 & 15 & Italia & $0.08 \%$ \\
\hline 14 & Eslovenia & 22.86 & & & & & & \\
\hline 15 & Eslovaquia & 21.57 & & & & & & \\
\hline 16 & Chipre & 14.82 & & & & & & \\
\hline 17 & Irlanda & 14.11 & & & & & & \\
\hline
\end{tabular}

a Medias del período 2000-2013 ordenadas a partir de los datos de la tabla 2

b Medias del período 2000-2013 ordenadas a partir de los datos de la tabla 3

c Variación porcentual entre las medias de los países

(Situada en el punto 4.3) 
A continuación, la tabla 5 compara la evolución seguida en cada país por ambos tipos, realizando las dos clasificaciones que se observan.

Tabla 5. Clasificación según reducción porcentual del período

\begin{tabular}{|c|l|r|r|l|r|}
\hline \multicolumn{3}{|c}{ Ranking TN } & \multicolumn{3}{c|}{ Ranking TIE } \\
\hline 1 & Chipre & $-56,90 \%$ & 1 & Bélgica & $-37,19 \%$ \\
\hline 2 & Irlanda & $-47,92 \%$ & 2 & Alemania & $-31,43 \%$ \\
\hline 3 & Alemania & $-41,47 \%$ & 3 & Países Bajos & $-28,03 \%$ \\
\hline 4 & Eslovenia & $-41,38 \%$ & 4 & Eslovaquia & $-26,72 \%$ \\
\hline 5 & Grecia & $-35,00 \%$ & 5 & Portugal & $-22,74 \%$ \\
\hline- & UEMb & $-28,99 \%$ & 6 & Finlandia & $-19,65 \%$ \\
\hline 6 & Países Bajos & $-28,57 \%$ & - & UEMb & $-18,85 \%$ \\
\hline- & UEMa & $-28,30 \%$ & - & UEM17b & $-17,51 \%$ \\
\hline- & UEM17b & $-26,48 \%$ & 7 & Italia & $-7,36 \%$ \\
\hline 7 & Austria & $-26,47 \%$ & 8 & Irlanda & $-6,42 \%$ \\
\hline- & UEM17a & $-24,58 \%$ & 9 & Francia & $-4,30 \%$ \\
\hline 8 & Italia & $-23,97 \%$ & 10 & Chipre & $-1,51 \%$ \\
\hline 9 & Luxemburgo & $-22,13 \%$ & 11 & Eslovenia & $2,46 \%$ \\
\hline 10 & Eslovaquia & $-20,69 \%$ & 12 & Grecia & $3,96 \%$ \\
\hline 11 & Estonia & $-19,23 \%$ & 13 & España & $7,03 \%$ \\
\hline 12 & Finlandia & $-15,52 \%$ & 14 & Austria & $24,51 \%$ \\
\hline 13 & Bélgica & $-15,42 \%$ & 15 & Luxemburgo & $34,57 \%$ \\
\hline 14 & España & $-14,29 \%$ & & & \\
\hline 15 & Portugal & $-10,51 \%$ & & & \\
\hline 16 & Francia & $-4,50 \%$ & & & \\
\hline 17 & Malta & $0,00 \%$ & & & \\
\hline & Ren & \\
\hline
\end{tabular}

a Reducciones porcentuales del $T N$ entre 2000 y 2013, ordenadas a partir de la tabla 2

b Reducciones porcentuales del TIE entre 2000 y 2013, ordenadas a partir de la tabla 3

(Situada en el punto 4.3)

Así, en ambas agrupaciones, se observa una diferencia media en torno a 10 puntos entre los recortes realizados en el tipo nominal - UEM17b (26,5\%) y UEMb $(29,0 \%)$ - frente a los del tipo efectivo - UEM17b $(17,5 \%)$ y $\operatorname{UEMb}(18,9 \%)$ - lo cual remarca en ambos casos la influencia de los incentivos fiscales.
En el caso de los países, Luxemburgo, Chipre, Austria, Eslovenia, Grecia e Irlanda son los que presentan mayor desigualdad entre la evolución de los tipos, habiendo recortado en un gran porcentaje el tipo nominal, aunque ello ha repercutido en el efectivo de manera muy leve. Esta discrepancia indica que los recortes del tipo estatutario han ido acompañados de reajustes en los incentivos y deducciones fiscales que han impedido la reducción de la tributación empresarial efectiva. En cambio, Bélgica, Portugal y Finlandia, presentan un recorte del tipo efectivo más pronunciado que en el tipo nominal, entre el $4 \%$ y el $22 \%$. Estos hechos reflejan que estos países han apostado por introducir incentivos y deducciones fiscales que, junto a las rebajas del tipo nominal, consiguen reducir la presión fiscal efectiva.

\section{Conclusiones}

Los resultados obtenidos en esta investigación remarcan que la evolución de la tributación empresarial seguida por la eurozona en los primeros catorce años desde su creación es cada vez más distante al comparar el tipo nominal y el tipo efectivo. Este hecho se corrobora en que (1) la tendencia descendente observada en el período es menor en el TIE que la seguida por el TN, (2) existe una mayor dispersión entre las medias del TIE que en las del TN, (3) los países no tienden a la homogeneización del impuesto sobre sociedades, pues este cada vez difiere más entre ellos causando grandes discrepancias a través de la utilización de los incentivos fiscales, (4) y se distinguen diferentes reducciones entre los países por la utilización o no de modificaciones en los incentivos fiscales que acompañan a los recortes en el TN.

La inclusión de los incentivos fiscales en las reformas delTN produce que los recortes en este tipo impositivo no se trasladen a la tributación empresarial efectiva. 
De ahí que la tendencia decreciente observada en el TN no se vea reflejada en el TIE, teniendo en cuenta la diversidad en materia de política fiscal empresarial que existe entre todos los países miembros.

En la actualidad, la UEM atraviesa grandes dificultades y además prevé un horizonte oscuro a corto plazo hasta que puedan aparecer los primeros datos positivos de crecimiento consolidado, sobre todo en los países más afectados por la crisis económica como Grecia, Portugal, España o Irlanda. En esta situación de incertidumbre, se siguen tomando medidas para eliminar la desconfianza en los mercados y una de las propuestas que se advierte en ideario de la Comisión Europea es la adopción de un impuesto sobre sociedades común para todos los Estados miembros.

Aunque en períodos distintos, el presente estudio corrobora los resultados obtenidos por Buijink y otros (2002), al comprobar que los incentivos fiscales existentes en los diferentes países, durante el período 2000-2013, no tienen un efecto homogeneizador en la presión fiscal empresarial, sino más bien todo lo contrario. Así pues, la importancia de las diferencias constatadas entre los diferentes países para el período analizado puede servir de referencia tanto en artículos relacionados, como para situar la posible armonización del impuesto sobre sociedades en su contexto actual, al poder observar la evolución de la presión fiscal efectiva en la zona euro en sus catorce primeros años de historia.

Por último, cabe señalar que los resultados deben tomarse con cierta cautela, debido a que la muestra utilizada solo considerada empresas cotizadas y la investigación se ha llevado a cabo utilizando la información de carácter financiero. Por lo tanto, hay que ser cautos al extrapolar a otros países o regiones geográficas.

\section{Referencias}

Álvarez, Santiago, Elena Fernández \& Antonio Martínez (2011). Corporate tax burden in the European Union. EC Tax Review, 1, 41-55.

Buijink, Willem, Boudewijn Janssenb \& Yvonne Schols (2002). Evidence of the effect of domicile on corporate average effective tax rates in the European Union. Journal of International Accounting, Auditing \& Taxation, 11, 115-130. https://doi.org/10.1016/ S1061-9518(02)00069-1

Collins, Julie H., Deen Kemsley \& Douglas A. Shackelford (1995). Tax reform and foreign acquisitions: A microanalysis. National Tax Journal, 48, 1-21.

Comisión Europea (2001). Una estrategia destinada a dotar a las empresas de una base imponible consolidada del impuesto sobre sociedades para sus actividades a escala comunitaria. COM (2001), 582 final.

Comité de Expertos Independientes sobre la Imposición de Sociedades (1992). Conclusiones y recomendaciones del comité de expertos independientes sobre imposición de sociedades. Informe Ruding. Monografía de Hacienda Pública Española, 2.

Comisión Europea (2011). Propuesta de directiva del consejo relativa a una base imponible consolidada común del impuesto sobre sociedades (BICCIS). COM (2001), 121 final.

Comisión Europea (2014). Taxation trends in European Union, data for the EU Member States, Iceland and Norway. Bruselas: Directorate General Taxation and Customs Union and Eurostat. Publications Office of the European Union.

Devereux, Michael P. \& Rachel Griffith (2003). Evaluating tax policy for location decisions. International Tax and Public Finance, 10, 107-126. https://doi. org/10.1023/A:1023364421914

Dyreng, Scott D., Michelle Hanlon \& Edward L. Maydew (2008). Long-run corporate tax avoidance. 
Accounting Review, 83(1). https://doi.org/10.2308/ accr.2008.83.1.61

Edgerton, Jesse (2010). Investment incentives and corporate tax asymmetries. Journal of Public Economics, 94(1112), 936-952. https://doi.org/10.1016/j.jpubeco. 2010.08.010

Elschner, Christina \& Werner Vanborren (2009). Corporate effective tax rates in an enlarged European Union. Taxation Papers 14. Bruselas: Directorate General Taxation and Customs Union, European Commission.

Hemmelgarn, Thomas \& Gaëtan Nicodème (2010). The 2008 financial crisis and taxation policy. Taxation Papers 20. Bruselas: Directorate General Taxation and Customs Union, European Commission.

Jacobs, Otto H. \& Christoph Spengel (2000). Measurement and development of the effective tax burden of companies: An overview and international comparison. International Tax Review, 28(10), 334-351.

Lazăr, Sebastian (2013). Effective corporate taxation in Romania. Evidence from the Bucharest Stock Exchange. Eastern European Economics, 51(4), 50-83. https://doi. org/10.2753/EEE0012-8775510403

Markle, Kevin S. \& Douglas A. Shackelford (2012). Cross-country comparisons of corporate income taxes. National Tax Journal, 65, 493-528. https://doi. org/10.17310/ntj.2012.3.01
Molina, Rafael (2005). Presión fiscal en las pymes: Estudio de su incidencia en la Comunidad Valenciana. Madrid: Aeca.

Nicodème, Gaëtan (2007). Comparing effective corporate tax rate. Frontiers in Finance and Economics, 4(2), 102-131.

Plesko, George A. (2003). An evaluation of alternative measures of corporate tax rates. Journal of Accounting and Economics, 35, 201-226. https://doi.org/10.1016/ S0165-4101(03)00019-3

Rohaya, Md Noor, Matsuki Nor'Azam \& Bardai Barjoyai (2008). Corporate effective tax rates: A study on Malaysian public listed companies. Malaysian Accounting Review, 7(1), 1-20. https://doi.org/10.7763/ ijtef.2010.v1.34

Romero, Esteban, Rafael Molina \& Gregorio Labatut (2009). La presión fiscal en las empresas espańolas: un estudio de las diferencias entre comunidades autónomas y sus efectos sobre las empresas de reducida dimensión. Revista Internacional de la Pequeña y Mediana Empresa, 1(2), 78-96.

Fecha de recepción: 16 de mayo de 2016 Fecha de aceptación: 05 de setiembre de 2016 Correspondencia: Rafael.Molina@uv.es tbarbera7@gmail.com 